\section{La educación energética favorecida desde el método explosivo valorativo.}

Energy education favored from the explosive valuation method.

Educação energética favorecida pelo método de avaliação explosiva.

Zaimar Domínguez Claro ${ }^{1}$, Paula Inés Reyes
Céspedes y Dayana Morales Parra
${ }^{1}$ Universidad de Holguín, Cuba
zaimar@uho.edu.cu
Recibido:06/01/2020

Aceptado: $13 / 05 / 2020$

Publicado: 30/06/2020

\title{
RESUMEN
}

El trabajo que se presenta es producto de una investigación realizada en la Universidad de Ciencias Pedagógicas de Holguín, dentro del Decenio de la Educación para el Desarrollo Sostenible, el cual proclama la necesidad de acciones educativas que contribuyan a solucionar los problemas energéticos. La propuesta elaborada pretende ofrecer una correcta orientación educativa para la educación energética desde las acciones dirigidas a la integración de los contenidos conceptuales, procedimentales y actitudinales, a partir del método explosivo valorativo que dinamiza la integración mediante situaciones educativas. Los resultados obtenidos a partir de métodos empíricos y teóricos forman parte de un proyecto de investigación y de una tesis de doctorado. La implementación de la propuesta en la carrera de licenciatura en Educación, especialidad Física; y la valoración de los resultados demostraron su efectividad sustentada en un preexperimento y un estudio de caso. Ello es un indicio fáctico de su valor práctico.

PALABRAS CLAVE: actitudes, conocimientos, educación energética, habilidades

\section{ABTRACT}

The work presented is the product of a research carried out at the Holguin University of Pedagogical Sciences, within the Decade of Education for Sustainable Development, which proclaims the need for educational actions that contribute to solving energy problems. The elaborated proposal pretends to offer a correct educational orientation for the energy education from the actions directed to the integration of the conceptual, procedural and attitudinal contents, from the explosive evaluative method that dynamizes the integration through educational situations. The results obtained from empirical and theoretical methods are part of a research project and a doctoral thesis. The implementation of the proposal in the Bachelor's degree in Education, Physical specialty; and the evaluation of the results demonstrated its effectiveness supported by a pre-experiment and a case study. This is a factual indication of its practical value.

KEYWORDS: attitudes, energy education, knowledge, skills 


\section{RESUMO:}

O trabalho apresentado é o produto de pesquisa realizada na Universidade de Ciências Pedagógicas de Holguin, na década de Educação para o Desenvolvimento Sustentável, que proclama a necessidade de ações educativas que contribuam para a solução de problemas energéticos. A proposta elaborada pretende oferecer uma orientação educacional correta para a educação energética a partir das ações direcionadas à integração dos conteúdos conceituais, procedimentais e atitudinais, a partir do método avaliativo explosivo que dinamiza a integração através de situações educacionais. Os resultados obtidos a partir de métodos empíricos e teóricos fazem parte de um projeto de pesquisa e de uma tese de doutorado. A implementação da proposta no curso de graduação em Educação Física; e a avaliação dos resultados demonstrou sua eficácia apoiada por um pré-experimento e um estudo de caso. Esta é uma indicação factual do seu valor prático.

PALAVRAS CHAVE: atitudes, conhecimento, educação energética, habilidades

\section{INTRODUCCIÓN}

La problemática del consumo energético y su implicación para el ser humano ha sido abordada en diversos encuentros a escala mundial. En 2018, la Asamblea General de las Naciones Unidas continúa proclamando a una Educación para el Desarrollo Sostenible, cuyas metas implican la acción de todos los educadores en la formación de una ciudadanía atenta a la situación del planeta, en la cual la educación energética contribuya a que las personas elijan estilos de vida que no socaven la base de recursos naturales, ni incidan de manera negativa en la equidad y justicia social de sus semejantes, para avanzar hacia un futuro sostenible (Organización de las Naciones Unidas para la Educación, la Ciencia y la Cultura, 2017).

En Cuba, la concepción de la carrera de licenciatura en Educación, especialidad Física, tiene a la educación energética como parte implícita de los problemas profesionales a los que se enfrenta el estudiante, razón por la que se contempla en uno de sus objetivos generales, el cual exige una educación orientada hacia la realización consciente de acciones contra la dilapidación de portadores energéticos y, con ello, contribuir a la calidad de vida de las personas; de allí su importancia para la trasmisión de modos de actuación responsables hacia el medio ambiente, a través del análisis de la situación energética actual, que ilustre la necesidad del uso sostenible de la energía como vía para el mejoramiento económico, social y ambiental.

El presente trabajo tiene como objetivo mostrar cómo ocurre el proceso de educación energética a partir de una propuesta contentiva de recursos pedagógicos, que logre la integración de contenidos conceptuales, procedimentales y actitudinales de la educación energética en los estudiantes de la carrera de licenciatura en Educación, especialidad Física, mediante el método explosivo valorativo.

\section{DESARROLLO}

La educación energética como parte de la educación ambiental tiene características e influencias propias provenientes del agravamiento, para la humanidad, de los problemas energéticos actuales (Ramos y Llanos, 2017), lo cual ofrece un camino contentivo de categorías relacionadas con la energía, su producción, uso, degradación y las relaciones que estos procesos guardan con el desarrollo sostenible. 
Domínguez (2012) acredita la necesaria integración de los contenidos de la educación energética y analizan las relaciones que, en el plano interno del alumno, determinan la posibilidad de tal integración, además enfatiza en que el logro de ese propósito implica a los alumnos y profesores, en la realización de acciones para contribuir a su desarrollo en los demás. En este sentido, hay coincidencia con Peña (2009), como se citó en Perera (2010), quien define la integración de contenidos como:

...proceso de carácter objetivo y subjetivo en que los sujetos cognoscentes al interactuar entre sí y con el objeto que estudian, desarrollan en el plano de lo externo distintos procederes que le permiten en el plano de lo interno, desde la actividad cognoscitiva, la apropiación de saberes integrados. (p. 82)

La variedad de los trabajos se verifica en la elaboración de estrategias, metodologías, tareas integradoras, entre otros, que por lo general atienden la formación de conceptos relacionados con la energía y su ahorro.

Desde la perspectiva teórica (Domínguez y Pérez, 2016) fundamenta los nexos entre los contenidos de la educación energética con los que se forman de manera espontánea en la vida cotidiana y en el ambiente escolar, aspecto relevante en la educación científica, y propone el método explosivo valorativo para la integración de los contenidos de la educación energética.

Un aspecto de singular importancia dentro de los fundamentos de la educación energética, ya establecidos, es la definición de este concepto. Los rasgos que se han sistematizado lo caracterizan adecuadamente, no obstante, se insiste en un rasgo que se considera importante y es: la necesidad de que todo ciudadano influya, con acciones concretas, en la educación energética de los otros, aspecto que se hace más significativo en los profesores, particularmente en los de Física.

En este sentido, Domínguez (2012) define que la educación energética es el proceso en que se dirige el intercambio sistemático entre sujetos y de estos con la naturaleza, dando lugar a la apropiación de conocimientos, habilidades y actitudes relacionadas con el uso sostenible de la energía, así como a la influencia en los demás para contribuir a la solución del problema energético global.

\section{El proceso de educación energética desde lo teórico metodológico}

El proceso pedagógico de la educación energética asume aquellos componentes que se concretan, de manera diferente de otras propuestas teórico-metodológicas previas: objetivos, contenidos y métodos de la educación energética.

\section{Objetivos de la educación energética}

Sus objetivos expresan el resultado al que se aspira; al lograrse, satisfacen la necesidad de la inserción del estudiante en la sociedad con una actitud transformadora.

Derivado de este propósito, de los objetivos de la carrera de Física y los objetivos propuestos por Pérez (2009) para la carrera de Física Electrónica, se hace la siguiente propuesta para la modalidad presencial de la licenciatura en Educación, especialidad Física: Demostrar con el ejemplo y actuación diarios la apropiación de conocimientos, habilidades y actitudes relacionados con los problemas energéticos globales y sus repercusiones ambientales, sociales y económicas en el marco del desarrollo sostenible, que le permita dirigir la educación energética de los educandos del nivel medio, para lo cual utilizará los recursos pedagógicos a su alcance en el cumplimiento de sus funciones profesionales. 
La derivación por años de este objetivo transita de exigencias argumentativas vinculadas a los contenidos de la educación energética, desde una perspectiva sostenible, a la realización de valoraciones relacionadas con los actuales problemas energéticos y su solución local; y de estas a la realización de acciones educativas encaminadas a la educación energética de los estudiantes de la escuela media cubana.

\section{Contenidos de la educación energética}

Al determinar los contenidos de la educación energética se tuvo en cuenta las insuficiencias presentes en su estado actual de desarrollo y los criterios del colectivo pedagógico del grupo de primer año. En este caso, se tomaron como contenidos los conocimientos relacionados con los actuales problemas energéticos, y los de tipo pedagógico, vinculados a la educación energética; las habilidades y las actitudes energéticas.

Los conocimientos comprenden los conceptos físicos enlazados con la energía y con los procesos que condicionan los problemas energéticos contemporáneos y su solución (energía, trabajo, potencia, cantidad de calor, eficiencia, temperatura, fuente de energía, fuente renovable de energía, fuente no renovable de energía, ley de conservación de la energía; producción, consumo y degradación y ahorro de energía), que se imbrican con otros de corte medioambiental (recursos naturales, cambio climático, contaminación ambiental, equidad y desarrollo sostenible).

A partir de la definición del concepto de educación energética se incluye este dentro del sistema de conocimientos y el de actitudes energéticas, que se complementan con los de métodos y procedimientos para la educación energética. Dentro del conjunto de habilidades que se requieren para desarrollarla, se jerarquizan las de argumentar y valorar, en particular esta última, porque requiere de conocimientos científicos para que se concrete, además de su nexo con la actividad valorativa, ligada a su vez con las actitudes.

Las actitudes energéticas son disposiciones estables de carácter orientador, determinadas integralmente por la situación existente y la experiencia anterior, que adopta el individuo hacia el ahorro de energía y hacia la obtención de información y la realización de acciones para influir en los demás sobre cómo solucionar los problemas energéticos locales.

Las actitudes energéticas se clasifican a partir de los rasgos esenciales de esta definición: disposición para el ahorro de energía, para la búsqueda y obtención de información y hacia la realización de acciones para influir en los demás con el fin de solucionar los problemas energéticos locales. Es importante destacar en este trabajo que las actitudes positivas favorecen los resultados en este tipo de actividad y las negativas son un indicador en la predicción del fracaso (Amérigo, García y Cortes, 2017).

\section{Métodos de la educación energética}

El proceso de educación energética forma sus contenidos mediante el método y estos son un aspecto fundamental para el logro de los objetivos. El método conduce a los estudiantes a involucrarse afectivamente en el proceso; garantiza la dirección de la actividad del estudiante, es decir, el orden y la secuencia de actividades que se realicen; y es la organización interna durante la ejecución del proceso. Este sistema de actividades que ejecutan el profesor y el estudiante se conoce como el procedimiento que hay que seguir para el desarrollo del método seleccionado.

El método explosivo valorativo consiste en el desarrollo de situaciones educativas que sorprendan, impacten y preocupen al estudiante, de manera que se cree en él un estado psíquico de desafío cognitivo y desestabilización actitudinal, surgido cuando un hecho conocido o un nuevo hecho se presenta contradictorio con sus conocimientos, vivencias y expectativas. La situación educativa 
descrita está asociada con los conocimientos y actitudes del estudiante, es decir, es una cuestión personológica que surge de la relación entre el objeto de aprendizaje y las condiciones internas del sujeto.

Su esencia radica en que la percepción global que tenía el estudiante de un objeto conocido cambia súbitamente. Lo inesperado provoca un estado emocional que lo priva de la reacción actitudinal acostumbrada y crea una situación problémica desde la perspectiva cognitiva.

El método concibe cómo orientar las actitudes que se desestabilizan (las espontáneas) en el sentido de las energéticas, proceso en el que se forman y fijan de manera integrada conocimientos y desarrollan habilidades específicas.

Establecida la concepción del método explosivo valorativo, se hace necesario determinar los procedimientos mediante los cuales él se implementa.

Procedimiento de diagnóstico de los estudiantes y selección o elaboración de actividades

- Procedimiento de orientación de las actividades

- Procedimiento de ayuda y consulta permanente

- Procedimiento de socialización y valoración de los resultados de la actividad

Los procedimientos en su conjunto conducen al desarrollo de la personalidad de los estudiantes como ciudadanos orientados al desarrollo sostenible, con habilidades profesionales que les permiten dirigir la educación energética de sus educandos.

Estrategia pedagógica para la educación energética en la modalidad presencial, de los estudiantes de licenciatura en Educación, especialidad Física.

Las estrategias pedagógicas son formas de dirección pedagógica que permiten la transformación del estado inicial del objeto hasta el deseado, y condiciona las acciones entre maestros y alumnos para alcanzar los objetivos.

Estas cuestiones condicionan la presencia de los siguientes elementos en la conformación de la estrategia para la educación energética: introducción, objetivo, direcciones, acciones, vías para la ejecución de las acciones y responsables.

\section{Introducción}

A partir del carácter complejo de la educación energética se toman el enfoque interdisciplinar como sustento de las acciones pedagógicas, y al colectivo pedagógico de grupo como estructura en la que dicho enfoque se concreta. Del currículo se toman las posibilidades que la disciplina Formación Laboral Investigativa en Enseñanza de la Física ofrece para desplegar el enfoque interdisciplinar y el método explosivo valorativo, de manera que:

- Durante el primer año los estudiantes se preparan en los ámbitos académico e investigativo para la práctica laboral.

- En segundo año, se incorporan a la práctica laboral en dos modalidades: sistemática y concentrada, diseño que se mantiene en el tercer año. 
Por tanto, el sistema de influencias se despliega en el contexto de la sede central universitaria y en la escuela de práctica laboral; por tanto, requiere acciones estratégicas de perfiles académico, investigativo y laboral.

\section{Objetivo general}

Desarrollar la educación energética en la modalidad presencial, de los estudiantes de la carrera de Física, a partir del accionar del colectivo pedagógico de grupo, mediante una concepción que integra el enfoque multidisciplinar y el interdisciplinar.

\section{Direcciones}

- Preparación del colectivo de profesores para el trabajo multi e interdisciplinar concebido para la educación energética.

- La educación energética de los estudiantes como ciudadanos.

- La preparación de los estudiantes como educadores en el área energético- ambiental.

Primera dirección: Preparación del colectivo de profesores para el trabajo multi e interdisciplinar concebido para la educación energética.

\section{Acciones}

- Profundización en el diagnóstico integral de los estudiantes mediante la aplicación de instrumentos que determinen sus conocimientos cotidianos y actitudes espontáneas.

- Elaboración de los indicadores de medida para el diagnóstico de los contenidos de la educación energética y los modos de evaluarla, con énfasis en los conocimientos cotidianos, la habilidad de valorar y las actitudes espontáneas y energéticas.

- Determinación de problemáticas de referencias relacionadas con la educación energética, que repercutan en la formación del educador que se necesita, plasmado en el Modelo del Profesional.

- Capacitación de los profesores en las exigencias del Modelo del Profesional y el Plan del Proceso Docente de la carrera Física, específicamente en lo relacionado con la educación energética: elaboración de tareas e implementación del método explosivo valorativo para la integración de los contenidos.

- Precisión de los objetivos generales de la asignatura mediante la derivación de los expresados en el Modelo del Profesional y la estrategia educativa de la carrera, atendiendo a los propósitos de la educación energética de la carrera.

- Planificación de actividades y tareas para la concreción del método explosivo valorativo en la integración de los contenidos de la educación energética.

- Diseño de actividades y tareas interdisciplinarias para la disciplina Formación Laboral Investigativa en Enseñanza de la Física, que favorezcan la utilización del método explosivo valorativo.

- Socialización de las experiencias individuales en la implementación de las acciones de educación energética. 
Vías: trabajo metodológico del colectivo pedagógico de grupo, trabajo científico metodológico de la carrera y autosuperación de los profesores.

Responsable: Jefe de carrera, profesores guías y profesores.

Segunda dirección: La educación energética de los estudiantes como ciudadanos.

\section{Acciones}

- Elaboración y desarrollo de un curso optativo electivo de educación energética para los estudiantes de la carrera de Física.

- Ejecución de tareas investigativas relacionadas con el medio ambiente en el contexto universitario y en la escuela de práctica, que conduzcan a la detección de problemas energéticos ambientales, sus causas y las vías de solución.

- Realización de tareas interdisciplinarias mediante la disciplina Formación Laboral Investigativa en Enseñanza de la Física, en las que se valoren problemáticas de la educación energética de los estudiantes de la escuela media cubana, sus causas y posibles vías de solución.

- Participación en competencias de habilidades, concursos y eventos científicos estudiantiles en los que se presenten los resultados de las tareas realizadas.

- Redacción de informes dirigidos a la dirección de la universidad y la escuela de práctica en los que se recojan los problemas ambientales detectados en esos contextos, con las causas detectadas y sugerencias para su solución o mejoramiento.

- Valoración de las actividades y tareas realizadas, así como de sus resultados.

Vía: la clase, la práctica laboral, las actividades extradocentes investigativas, el trabajo científico estudiantil y el trabajo metodológico de la carrera.

Responsable: Jefe de departamento, Jefe de carrera, jefes de disciplinas, profesores guías, profesores, estudiantes.

Tercera dirección: La preparación de los estudiantes como educadores en el área energéticoambiental.

\section{Acciones}

- Valoración del papel de la biblioteca y de las tecnologías de la informática en la universidad y en la escuela, para la obtención de información relacionada con los problemas energéticos actuales y su posible solución.

- Formación del sistema de conocimientos científicos y metodológicos previstos en el Modelo del Profesional, como sustento de la educación energética.

- Lograr un dominio meta cognitivo del estado de la formación de los contenidos de la educación energética mediante el estudio de las vías para realizar la educación energética en la escuela media cubana.

- Diagnóstico de conocimientos, habilidades y actitudes jerarquizando, mediante la observación, las actitudes que se manifiestan en el contexto de la práctica laboral.

- Identificación de problemas educativos de tipo energético, principalmente en el entorno de la escuela de práctica laboral. 
- Diseño de tareas investigativas proyectadas hacia la solución de problemas educativos de tipo energético ambientales para asignarlas a los estudiantes de la escuela media cubana.

- Diseñar, organizar y ejecutar acciones educativas de tipo energético en las escuelas de práctica.

- Socializar los resultados obtenidos en la planificación y dirección de acciones de educación energética en la escuela de práctica laboral.

Vía: la clase, la disciplina Formación Laboral Investigativa en Enseñanza de la Física, el trabajo científico estudiantil y visitas a la práctica laboral.

Responsables: jefes de disciplinas, profesores de Formación Pedagógica General, profesores guías, tutores, profesores y estudiantes.

\section{Concepción de la implementación de la estrategia}

La puesta en práctica de la estrategia está prevista en dos etapas. La primera prepara las condiciones para el trabajo interdisciplinar, garantiza la formación del sistema de conocimientos fundamentales relacionados con la energía y las relaciones Ciencia-Tecnología-Sociedad-Ambiente. Esta etapa corresponde al primer año de la carrera.

El método explosivo valorativo se aplica con una concepción disciplinar en las asignaturas que forman el sistema de conocimientos científicos, y mantiene una intencionalidad común: integrar conocimientos, la habilidad valorar y actitudes.

La segunda etapa está ubicada en el segundo y tercer años de la carrera, y se caracteriza por el accionar interdisciplinar del colectivo pedagógico, que abarca la elaboración y asignación de tareas para la práctica laboral investigativa y el trabajo científico estudiantil, parejo con la asignación de tareas desde las asignaturas. El método explosivo valorativo potencia, con la participación de cada vez más asignaturas, y mediante el trabajo interdisciplinar, la formación de los contenidos previstos.

Los estudiantes implementan acciones sistémicas para la educación energética de adolescentes y jóvenes e inician su labor de asesoría de los colectivos de las escuelas.

\section{Análisis y valoración de los resultados}

Para valorar la efectividad de la estrategia elaborada se recurrió a un preexperimento pedagógico de un grupo pre y pos test y a un estudio de caso.

La muestra fue intencional, de 21 estudiantes que en este caso coincide con la población. La variable independiente es la implementación de la estrategia pedagógica y la dependiente es la educación energética de los estudiantes, que contiene tres dimensiones: conocimientos científicos energéticos, la habilidad valorar y las actitudes energéticas, según la Tabla 1.

Cada dimensión y la variable fueron sometidas a una prueba de hipótesis (rangos señalados de Willcoxon), realizada mediante el SPS versión 15.0 para Windows. El diagnóstico de la educación energética de los estudiantes se obtuvo a partir de la suma de los indicadores establecidos para cada una de las dimensiones de la variable.

Mediante ese procedimiento, cada estudiante puede obtener puntuaciones entre (-2) y (9) puntos, con lo cual queda establecida una escala ordinal con 12 valores posibles y cuatro grados de formación de la educación energética (tabla 1 ). 
Tabla 1. Indicadores de la educación energética de los estudiantes.

\begin{tabular}{cccccc}
\hline CCE & HV & AE & PR & EE & CRITERIO \\
\hline 0 & 0 & -2 & {$[-2 ;-1 ; 0]$} & Muy Bajo & 1 \\
1 & 1 & -1 & {$[1 ; 2 ; 3]$} & Bajo & 2 \\
2 & 2 & 0 & {$[4 ; 5 ; 6]$} & Aceptable & 3 \\
3 & 3 & 1 & {$[7 ; 8 ; 9]$} & Alto & 4 \\
& 4 & 2 & & & \\
\hline
\end{tabular}

Nota: CCE: conocimientos científicos energéticos, $H V$ : la habilidad valorar y AE: actitudes energéticas.

Fuente: Elaboración propia.

El análisis de los grados de avance de la educación energética arroja que un estudiante (4.8\%) obtiene avance nulo, diecisiete $(81 \%)$ avanzan un grado y tres $(14.3 \%)$ avanzan dos grados. De los cinco estudiantes que inicialmente manifiestan un grado muy bajo de formación de la educación energética, cuatro avanzan al inmediato (bajo) y uno al aceptable. De los 13 estudiantes, al inicio, que tienen un grado bajo de formación de la educación energética, uno no avanza, 10 avanzan al inmediato superior (aceptable) y uno al grado alto de formación de la educación energética. Los tres estudiantes que al inicio muestran un grado aceptable de la educación energética avanzan al grado alto.

Para demostrar la significación de esos avances se usó la prueba de los rangos señalados de Willcoxon, con la intención de demostrar que el estado final de formación de la educación energética es superior a la inicial.

Se realizó además un estudio de caso que permite ahondar en los aspectos cualitativos del proceso pedagógico y tiene la finalidad de profundizar en cómo los estudiantes asumen y realizan las tareas que se les asignan e interpretan los resultados que obtienen, como vía para valorar la efectividad del método explosivo valorativo. Su objetivo se circunscribe a encontrar evidencias fácticas del efecto de las tareas

La triangulación de la información obtenida mediante el preexperimento pedagógico y el estudio de caso conduce a los siguientes hechos: se demuestra que las tareas docentes elaboradas, si se orientan, resuelven y socializan sus resultados mediante el método explosivo valorativo favorecen las condiciones para que se produzca la problematización cognitiva, la desestabilización de las actitudes espontáneas y la estabilización actitudinal en el sentido deseado, procesos que se producen de manera unida. El método explosivo valorativo propicia también la orientación necesaria para estimular el desarrollo de los escolares, en particular la formación de conocimientos científicos, la habilidad valorar y las actitudes energéticas.

Otro hecho importante es la necesidad de atender las diferencias individuales, pues si bien se manifiestan regularidades en los estudiantes sometidos al estudio de caso, se aprecian diferencias sensibles y notables tanto en las limitaciones como en las potencialidades de los estudiantes. Particular atención requiere los estudiantes que inicialmente poseen mayores limitaciones cognoscitivas y actitudinales.

\section{CONCLUSIONES}

El uso de métodos del nivel teórico y una contribución relacionada con la teoría pedagógica de la educación energética, condujo a las siguientes conclusiones:

La solución completa de los actuales problemas energéticos no se logra sin la concienciación de todos los ciudadanos acerca de esas problemáticas, sus causas y las alternativas de solución, razón por la que se requiere que los estudiantes de la carrera de Física se impliquen en la realización de acciones para 
contribuir a la educación energética de sus alumnos, al punto de que estos se ocupen de influir en los demás acerca de la necesidad de solucionar los mencionados problemas.

El método explosivo valorativo dinamiza la integración de los contenidos de la educación energética mediante situaciones educativas, que crean en los estudiantes un estado psíquico de desafío cognitivo y desestabilización actitudinal y facilita las condiciones para la posterior estabilización de las actitudes energéticas, la formación de conocimientos científicos y el desarrollo de habilidades.

La estrategia elaborada contiene recursos pedagógicos que se implementan mediante acciones dirigidas a la integración de los contenidos conceptuales, procedimentales y actitudinales previstos.

La triangulación de la información obtenida mediante el preexperimento pedagógico y el estudio de caso conducen a evidencias de la validez del método explosivo valorativo y sus procedimientos.

\section{REFERENCIAS BIBLIOGRÁFICAS}

Amérigo, M., García J.A., y Côrtes, P.L. (2017). Análisis de actitudes y conductas pro-ambientales: un estudio exploratorio con una muestra de estudiantes universitarios brasileños. Ambiente \& Sociedade, 20(3), 1-20. https://doi.org/10.1590/1809-4422asoc300r1v2032017

Domínguez, Z. (2012). La educación energética de los estudiantes de la carrera de Licenciatura en educación, especialidad Matemática Física [Tesis doctoral, Universidad de Ciencias Pedagógicas José de la Luz y Caballero]. Repositorio Institucional. https://cutt.ly/ZiqldQ2

Domínguez, Z., y Pérez, N. (2016). Los conocimientos cotidianos alternativos dentro de la educación Energética en la carrera Matemática Física. Revista Luz, 14(2). https://url2.cl/RyrHz

Organización de las Naciones Unidas para la Educación, la Ciencia y la Cultura. (2017). La UNESCO avanza la Agenda 2030 para el Desarrollo Sostenible. https://url2.cl/AJQnl

Perera, F. (2010). Pensamiento complejo, interdisciplinariedad y cultura científica. En C. Sifredo y N. Pupo (Comp.), Didáctica de las ciencias: nuevas perspectivas (pp.76-87). Editorial Educación Cubana. https://cutt.ly/TuvBsoD

Pérez, E. (2009). La superación profesional para la educación energética de profesores de los institutos superiores pedagógicos [Tesis doctoral, Universidad de Ciencias Pedagógicas José de la Luz y Caballero]. Repositorio Institucional. https://url2.cl/W5N7F

Ramos, J.L., y Llanos, M. (Comp.). (2017). Cultura energética desde la escuela. Editorial Universidad del Norte. https://url2.cl/b4Lfs 